\title{
Analysis of Nonlinear Traveling Waves by Frequency-Domain Perturbation Method
}

\author{
Akio USHIDA, Yuichi TANJI and Yoshifumi NISHIO
}

\author{
Dept. of Electrical and Electronic Engineering, Tokushima University \\ 2-1, Minami-Josanjima, Tokushima, 770 Japan \\ Tel. +81-886-56-7469 Fax. +81-886-56-7471 \\ E-mail ushida@ee.tokushima-u.ac.jp
}

\begin{abstract}
We discuss a numerical method for solving the nonlinear transmission lines by the frequency-domain perturbation method. To improve the convergence, we introduce two new methods of the compensation and the homotopy techniques, which also help to make the iteration stable. This kind of transmission lines is widely used in the communication curcuits such as GaAs integrated circuits, and varactor diode circuits.
\end{abstract}

\section{INTRODUCTION}

Nonlinear devices such as GaAs nonlinear transmission lines [1], varactor diodes and superconducting transmission lines [2] have the nonlinear capacitors. The velocity of traveling wave is given by

$$
v=1 / \sqrt{L(i) C(v)}
$$

so that if the capacitor is a decreasing function of the voltages, it will have an interesting property such that the higher part of the waveform is the faster than the lower part of it. The property is used in many purposes such as the picosecond pulse compression [1], picosecond shock-wave generations [3] and so on.

There are some papers discussing the phenomena from the physical points of view [1-5]. In reference [6], the shock-waves of nonlinear transmission line driven by a sinusoidal generator are analyzed by the harmonic balance method, where the nonlinear transmission line is replaced by the finite number of the discrete lumped models. The reference [7] has analyzed the phenomena by the application of the difference approximation to the nonlinear partial differential equation. In reference [8], they have analyzed the nonlinear wave propagation phenomena, where the 2-dimensional FFT is applied to solve the circuit equation in the distance and time.

In this paper, we discuss an efficient numerical method for solving the nonlinear transmission lines. The waveform is approximated by the Fourier expansion at each point on the line, and the coefficients are calculated by a perturbation technique. Although, the method is only applied to the weakly nonlinear systems, we have improved it with two techniques of $a$ compensational and homotopy method.

\section{FREQUENCY-DOMAIN PERTURBATION METHOD}

2.1. Perturbation Method Now, consider a nonlinear transmission line terminated by linear subnetworks. The circuit equation is described by the following nonlinear partial differential equations:

$$
-\frac{\partial v}{\partial x}=\frac{\partial \phi_{L}}{\partial t}+v_{R}, \quad-\frac{\partial i}{\partial x}=\frac{\partial q_{C}}{\partial t}+i_{G}
$$

where $\phi_{L}$ and $q_{C}$ are the flux of a nonlinear inductor and the charge of a nonlinear capacitor. We assume the characteristics as follows:

$$
\begin{gathered}
i_{L}=\Gamma \phi_{L}+\epsilon \hat{i}_{L}\left(\phi_{L}\right), \quad v_{R}=R i_{R}+\epsilon \hat{v}_{R}\left(i_{R}\right) \\
v_{C}=S q_{C}+\epsilon \hat{v}_{C}\left(q_{C}\right), \quad i_{G}=G v_{G}+\epsilon \hat{i}_{G}\left(v_{G}\right)
\end{gathered}
$$

where $\epsilon$ means a small constant, and $\hat{i}_{L}\left(\phi_{L}\right), \hat{v}_{R}\left(i_{R}\right)$, $\hat{v}_{C}\left(q_{C}\right)$ and $\hat{i}_{G}\left(v_{G}\right)$ are nonlinear terms. We have the relations of $i \equiv i_{L}=i_{R}$ and $v \equiv v_{C}=v_{G}$.

To analyze the traveling-waves, consider the responses of nonlinear transmission lines driven by an impulse waveform as follows:

$$
\begin{aligned}
e(t) & =E_{m} \sin \left(\frac{2 k \pi}{T} t\right) & & : 0 \leq t \leq \frac{T}{2 k} \\
& =0 & & : \frac{T}{2 k} \leq t \leq T
\end{aligned}
$$

where $T$ is a period of the impulse and $k$ is an integer. Assume that the nonlinear transmission line is terminated by a linear impedance $Z_{L}$. Then, the circuit equations (1) and (2) are mathematically reduced to the nonlinear partial equations constrained by a linear boundary condition. In this paper, we apply the frequency-domain approach, and describe the input impulse waveform by the Fourier expansion in the complex domain as follows:

$$
e(t)=\sum_{k=-M}^{M} E_{k} e^{j k \omega t}, \quad \omega=\frac{2 \pi}{T}
$$


for a sufficiently large $M$. Now, let us assume the solutions at the $m$ th iteration as follows:

$v^{m}(x, t)=\sum_{k=-M}^{M} V_{k}^{m} e^{j k \omega t}, \quad i^{m}(x, t)=\sum_{k=-M}^{M} I_{k}^{m} e^{j k \omega t}$

$\phi_{L}^{m}(x, t)=\sum_{k=-M}^{M} \Phi_{k}^{m} e^{j k \omega t}, \quad q_{C}^{m}(x, t)=\sum_{k=-M}^{M} Q_{k}^{m} e^{j k \omega t}$

Then, we have the following perturbed equations:

$$
\begin{aligned}
\frac{d V_{k}^{m}}{d x}= & -(R+j k \omega L) I_{k}^{m} \\
& -\epsilon\left(\hat{V}_{R, k}\left(i^{m-1}\right)-j k \omega L \hat{I}_{L, k}\left(\phi_{L}^{m-1}\right)\right)( \\
\frac{d I_{k}^{m}}{d x}= & -(G+j k \omega C) V_{k}^{m} \\
& -\epsilon\left(\hat{I}_{G, k}\left(v^{m-1}\right)-j k \omega C \hat{V}_{C, k}\left(q_{C}^{m-1}\right)\right)(
\end{aligned}
$$

where $L=1 / \Gamma, C=1 / S$ and

$$
\begin{aligned}
\Phi_{k}^{m} & =L\left(I_{k}^{m}-\epsilon \hat{I}_{L, k}\left(\phi_{L}^{m-1}\right)\right) \\
Q_{k}^{m} & =C\left(V_{k}^{m}-\epsilon \hat{V}_{C, k}\left(q_{C}^{m-1}\right)\right)
\end{aligned}
$$

Observe that the relations (6.1) and (6.2) are ordinary differential equation, and the second terms at the right hand side correspond to the forced terms calculated at $(m-1)$ th iteration. The solutions of $(6.1)$ and (6.2) are written as follows:

$$
\begin{aligned}
V_{k}^{m}(x) & =A_{k}^{m} e^{\lambda_{k} x}+B_{k}^{m} e^{-\lambda_{k} x}+\epsilon \hat{V}_{k}^{m}(x) \\
I_{k}^{m}(x) & =\frac{1}{Z_{k, 0}}\left(-A_{k}^{m} e^{\lambda_{k} x}+B_{k}^{m} e^{-\lambda_{k} x}\right)+\epsilon \hat{I}_{k}^{m}(x)
\end{aligned}
$$

where the propagation constant $\lambda_{k}$ and characteristic impedance $Z_{k, 0}$ are given by

$\lambda_{k}=\sqrt{(j k \omega L+R)(j k \omega C+G)}, \quad Z_{k, 0}=\sqrt{\frac{j k \omega L+R}{j k \omega C+G}}$

The first two terms of ( 7 ) correspond to the zero-input responses, and $\epsilon \hat{V}_{k}^{m}(x)$ and $\epsilon \hat{I}_{k}^{m}(x)$ to the zero-state responses calculated by a numerical intergration technique. The constant parameters $A_{k}^{m}$ and $B_{k}^{m}$ in (7) are evaluated by the boundary conditions of the transmission line. Thus, all of the Fourier coefficients $V_{k}^{m}$ and $I_{k}^{m}$ are caluculated, and $\Phi_{k}^{m}$ and $Q_{k}^{m}$ are decided by (6.3) and (6.4). The iteration is continued until the variation

$$
\delta^{m} \equiv\left[\sum_{k=-M}^{M}\left(V_{k}^{m}(l)-V_{k}^{m-1}(l)\right)^{2}\right.
$$

$$
\left.+\sum_{k=-M}^{M}\left(I_{k}^{m}(l)-I_{k}^{m-1}(l)\right)^{2}\right]^{1 / 2}
$$

becomes sufficiently small for given a constant $\delta$. However, we can not say anything whether the solution waveform is sufficiently accurate or not.

Now, let us define the following residual error. The solutions for the $M$ frequency components be $v_{M}(x, t)$, $i_{M}(x, t)$. Then, define the residual error as follows:

$$
\begin{aligned}
\varepsilon_{M} \equiv & {\left[\frac { 1 } { T } \int _ { 0 } ^ { T } \left\{\left(v_{M}(x, t)-\hat{v}(x, t)\right)^{2}\right.\right.} \\
& \left.\left.+\left(i_{M}(x, t)-\hat{i}(x, t)\right)^{2}\right\} d t\right]^{1 / 2}
\end{aligned}
$$

where $\hat{v}(x, t), \hat{i}(x, t)$ are the exact solutions, and $v_{M}(x, t), i_{M}(x, t)$ are the approximate solutions at $m$ th iteration with $M$ frequency components. Since it is impossible to get the exact solutions in (9), we assume the solutions obtained with the larger frequency components $M^{\prime}$ as the exact solutions.

The residual error (9) between the solutions with $M$ and $M^{\prime}$ frequency components are given by

$$
\begin{aligned}
\varepsilon_{M} \cong & {\left[\sum_{|k|=M}^{M^{\prime}}\left(V_{k}^{m}(l)-V_{k}^{m-1}(l)\right)^{2}\right.} \\
& \left.+\sum_{|k|=M}^{M^{\prime}}\left(I_{k}^{m}(l)-I_{k}^{m-1}(l)\right)^{2}\right]^{1 / 2}
\end{aligned}
$$

If the residual error $\varepsilon_{M}$ is not so small, we need to choose much larger $M$ for the waveform approximation, and again repeat our perturbation method.

2.2. Modified Perturbation Method It is said that the perturbation method is only applied to the weakly nonlinear circuits. In this section, we will modify the method such that it can be applied to the much stronger nonlinear circuits. Assume that the nonlinear characteristics given in (2) are monotone increasing functions, and for simplicity, describe it as follows:

$$
u_{1}=H u_{2}+\epsilon \hat{h}\left(u_{2}\right), H=\operatorname{diag}(\Gamma, R, S, G)
$$

where $u_{1}=\left(i_{L}, v_{R}, v_{C}, i_{G}\right)^{T}, \quad u_{2}=\left(\phi_{L}, i_{R}, q_{C}, v_{G}\right)^{T}$ and $\epsilon \hat{h}\left(u_{2}\right)=\epsilon\left(\hat{i}_{L}\left(\phi_{L}\right), \hat{v}_{R}\left(i_{R}\right), \hat{v}_{C}\left(q_{C}\right), \hat{i}_{G}\left(v_{G}\right)\right)^{T}$. Since the convergence of our perturbation method largely depens on the magnitude of perturbed terms $\epsilon \hat{h}\left(u_{2}\right)$, we need to decrease it compared with the linear term. Thus, we introduce small constant

$$
\Delta H \equiv \operatorname{diag}(\Delta \Gamma, \Delta R, \Delta S, \Delta G)
$$


as a compensation parameter in the following form:

$$
u_{1}=(H+\Delta H) u_{2}+\left(\epsilon \hat{h}\left(u_{2}\right)-\Delta H u_{2}\right)
$$

The second term is the nonlinear function corresponding to the perturbed term which can be be largely decreased by choosing a suitable $\Delta H$. We found from our numerical examples that the convergence ratio is largely improved by introducing of the compensation parameter.

Homotopy method is sometimes used when the iteration method does not guarantee the convergency. We will apply it to our perturbation method. Let us introduce a parameter $\rho$ for the nonlinear elements (2).

$$
\begin{gathered}
u_{1}=(H+\Delta H) u_{2}+\rho\left(\epsilon \hat{h}\left(u_{2}\right)-\Delta H u_{2}\right), \\
\rho: 0 \longrightarrow 1
\end{gathered}
$$

It is clear that the relation (12) for $\rho=0$ corresponds to linear elements, and the nonlinearity is gradually increased by choosing $\{\rho: 0 \longrightarrow 1\}$. At $\rho=1$, it reduces to the original function. If we choose a small variation $\Delta \rho$ per iteration such as

$$
\rho^{m}=\rho^{m-1}+\Delta \rho
$$

our perturbation can also get the solution stablely.

2.3. Convergence Condition Now, let us consider the convergence condition of our algorithm. For simplicity, let us introduce the following symbols for variables in (6) and (7):

(a). For the solutions, we set

$$
X_{1, k}^{m}(x)=\left(\begin{array}{c}
V_{k}^{m}(x) \\
I_{k}^{m}(x)
\end{array}\right), X_{2, k}^{m}(x)=\left(\begin{array}{c}
\Phi_{k}^{m}(x) \\
Q_{k}^{m}(x)
\end{array}\right)
$$

(b). For the perturbed terms, we set

$$
\begin{aligned}
& \hat{X}_{1, k}\left(w_{1}^{m-1}, x\right)=\left(\begin{array}{c}
\hat{V}_{R, k}\left(i^{m-1}\right) \\
\hat{I}_{G, k}\left(v^{m-1}\right)
\end{array}\right) \\
& \hat{X}_{2, k}\left(w_{2}^{m-1}, x\right)=\left(\begin{array}{c}
\hat{I}_{L, k}\left(\phi_{L}^{m-1}\right) \\
\hat{V}_{C, k}\left(q_{C}^{m-1}\right)
\end{array}\right)
\end{aligned}
$$

where $w_{1}=(v, i)^{T}, w_{2}=\left(\phi_{L}, q_{C}\right)^{T}$.

Set the coefficient matrices of (6) as follows:

$$
\begin{gathered}
A_{k}=\left(\begin{array}{cc}
0 & R+j k \omega L \\
G+j k \omega C & 0
\end{array}\right) \\
B_{k}=\left(\begin{array}{cc}
j k \omega L & 0 \\
0 & j k \omega C
\end{array}\right), \quad C_{0}=\left(\begin{array}{cc}
0 & L \\
C & 0
\end{array}\right)
\end{gathered}
$$

Now, describe the zero-state response of (7) in the integration form.

$$
\begin{aligned}
& X_{1, k}^{m}(x)=C_{1, k}(x) E_{k}+C_{2, k}(x) X_{1, k} X_{1, k}^{m}(l) \\
&-\epsilon \int_{0}^{x} e^{-A_{k}(x-s)} \hat{X}_{1, k}\left(w_{1}^{m-1}, s\right) d s \\
&-\epsilon B_{k} \int_{0}^{x} e^{-A_{k}(x-s)} \hat{X}_{2, k}\left(w_{2}^{m-1}, s\right) d s \\
& X_{2, k}^{m}(x)=C_{0} X_{1, k}^{m}(x)-\epsilon C_{0} \hat{X}_{2, k}\left(w_{2}^{m-1}, x\right)
\end{aligned}
$$

where $C_{1, k}(x), C_{2, k}(x)$ are decided from the boundary conditions and (7).

Let us adopt the following norm

$$
\|w\| \equiv \sqrt{\frac{1}{T} \int_{0}^{T} w^{2}(t) d t}
$$

Then, we have the following convergence conditions. Theorem: Assume that, for an approximate solution $\left(w_{1}^{0}, w_{2}^{0}\right)^{T}$, there are constants $D_{1}, D_{2}, K_{0}, L_{1}$ and $L_{2}$ satisfying as follows:

(i) Define the solution domain by:

$$
\begin{gathered}
\Omega \equiv\left\{w_{1}, w_{2} \mid\left\|w_{1}-w_{1}^{0}\right\| \leq D_{1},\right. \\
\left.\left\|w_{2}-w_{2}^{0}\right\| \leq D_{2}\right\}
\end{gathered}
$$

(ii) Define norms of the coefficient matrices by:

$$
\left\|C_{0}\right\| \equiv K_{0}, \quad K_{1} \equiv \max _{-M \leq k \leq M} \frac{\left\|A_{k}^{-1}\right\| L_{1} l}{1-C_{2 k}}
$$

$$
K_{2} \equiv \max _{-M \leq k \leq M} \frac{\left\|B_{k} A_{k}^{-1}\right\| L_{2} l}{1-C_{2 k}}
$$

where

$$
C_{2 k} \equiv \max _{0 \leq x \leq l}\left\|C_{2, k}(x)\right\|
$$

(iii) Assume the perturbed terms satisfy the following Lipschitz conditions:

$$
\begin{aligned}
& \left\|\hat{X}_{1}\left(w_{1}^{\prime}, x\right)-\hat{X}_{1}\left(w_{1}^{\prime \prime} 0, x\right)\right\| \leq L_{1}\left\|w_{1}^{\prime}-w_{1}^{\prime \prime}\right\| \\
& \left\|\hat{X}_{2}\left(w_{2}^{\prime}, x\right)-\hat{X}_{2}\left(w_{2}^{\prime \prime}, x\right)\right\| \leq L_{2}\left\|w_{2}^{\prime}-w_{2}^{\prime \prime}\right\|
\end{aligned}
$$

for all $0 \leq x \leq l$, where $L_{1}$ and $L_{2}$ are Lipschitz constants.

(iv) Set the maximum values of the variables in $x=[0, l]$ as follows:

$X_{1, k}^{m}=\max _{0 \leq x \leq l}\left\|X_{1, k}^{m}(x)\right\|, X_{2, k}^{m}=\max _{0 \leq x \leq l}\left\|X_{2, k}^{m}(x)\right\|$ 
Furthermore, if it satisfies the condition,

$$
\begin{gathered}
\kappa \equiv \epsilon\|P\|<1 \\
\text { for } P=\left(\begin{array}{cc}
K_{1} & K_{2} \\
K_{0} K_{1} & K_{0} L_{2}\left(K_{2}+L_{2}\right)
\end{array}\right)
\end{gathered}
$$

our perturbation method (13) will converge to the unique solution $\left(w_{1}^{M}, w_{2}^{M}\right)^{T}$.

We found from the theorem that our perturbational iteration will be converged to an approximate solution $X^{M}(l)$ if the nonlinear term $\epsilon$ is small.

\section{ILLUSTRATIVE EXAMPLES}

As an example of the nonlinear transmission line, let us consider to analyze the shock-wave to an impulse response. Assume the transmission line is terminated by a resistor $R_{L}=10$ and

$$
\begin{gathered}
i_{L}=350 \times 10^{11} \phi_{L}, \quad v_{R}=0.015 i_{R}+0.1 i_{R}^{3} \\
v_{C}=10^{11} q_{C}+0.5 \times 10^{34} q_{C}^{3}, \quad i_{G}=0.015 v_{G}+0.1 v_{G}^{3}
\end{gathered}
$$

For the input impulse, we choose $E_{m}=0.25, T=1$ and $k=16$ in (3). We found from the example that our perturbation method is largely improved by the combinations of compensation method and homotopy method.

\section{CONCLUSIONS AND REMARKS}

We have presented a frequency-domain perturbation algorithm for calculating transient responses of a nonlinear transmission lines. We can largely improve the convergence by introducing two methods of the compensation method and homotopy method.

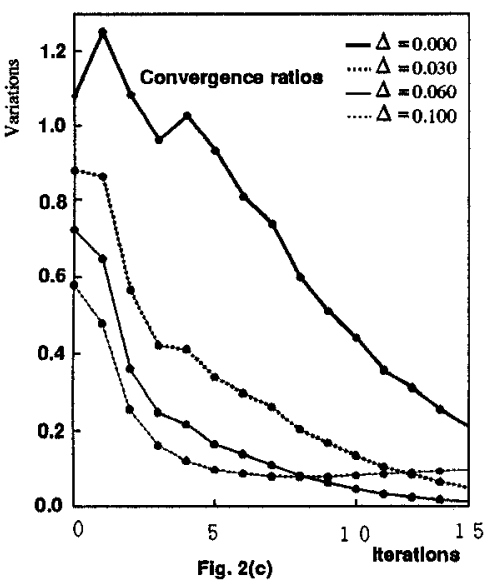

Figure 1: Convergence ratios for various compensational parameter, where variation

$$
\delta_{m}=\left\|X^{m}(l)-X^{m-1}(l)\right\|
$$

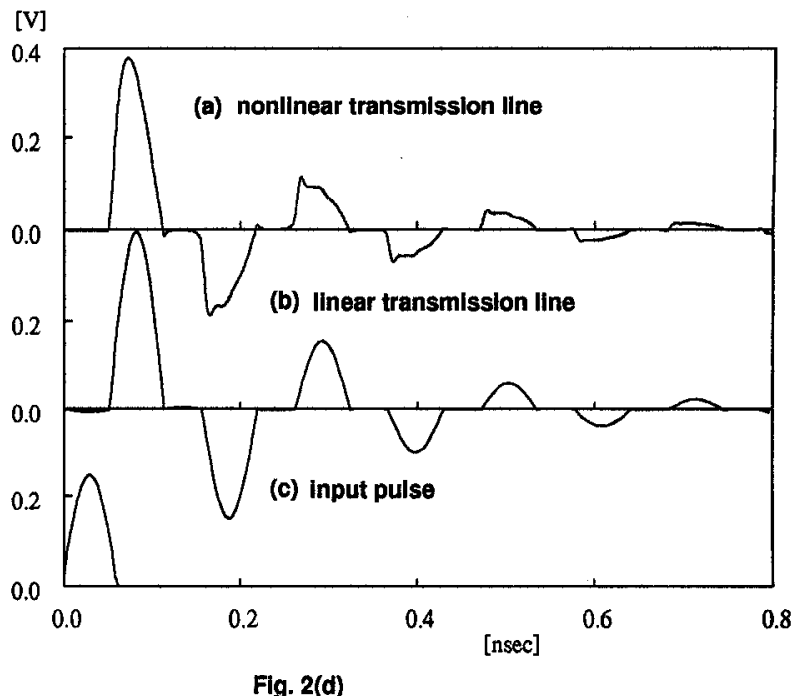

Figure 2: Impulse responses

(a) The response of nonlinear transmission line (b) The response of linear transmission line where nonlinear terms are set to be zeros (c) Input impulse

\section{REFERENCES}

[1] M.J.W. Rodwell, "Nonlnear transmission line for picosecond pulse compression and broadband phase modulation," Electron. Lett.,vol.23, no.3, pp.109110,1987

[2] R.K. Arora and J.L. Thaker, "Electromagnetic pulse transmission on superconducting interconnects", Int. J. Electronics, vol.71, no.1, pp.117-125, 1991

[3] C.J. Madden, R.A. Marsland, M.J.W. Rodwell, D.M. Bloom and Y.C. Pao, "Hyperabrupt-doped GaAs nonlinear transmission line for picosecond shock-wave generation", Appl. Phys. Lett., vol.54, no.11, pp.1019-1021, 1989

[4] R. Landauer, "Shock waves in nonlinear transmission lines and their effect on parametric amplification", IBM Journal, October, pp.391-401, 1960

[5] D. Jager and F-J. Tegude, "Nonlinear wave propagation along periodic-loaded transmission line", Appl. Phys., vol.15, pp.393-397, 1978

[6] C. Camacho-Penalosa and I. Molina-Fernandez, "Harmonic balance analysis of nonlinear transmission lines", Electron. Lett., vol.24, no.9, pp.12351236,1988

[7] R.H. Freeman and A.E. Karbowiak, "An investigation of nonlinear transmission lines and shock waves", J. Phys. D: Appl. Phys,, vol.10, pp.633643, 1977

[8] I. Molina-Fernandez, C. Camacho-Penalosa and J. Ramos, "Application of the two-dimensional Fourier transform to nonlinear wave propagation phenomena", IEEE Trans. on Microwave Theory Tech., vol.42, no.6, pp.1079-1085, 1994 